\title{
Solvability of second order linear differential equations in the sequence space $n(\phi)$
}

\author{
Abdullah Alotaibi ${ }^{1}$, M. Mursaleen ${ }^{1,2^{*}}$ and Badriah A.S. Alamri
}

\section{"Correspondence:}

mursaleenm@gmail.com

1 Operator Theory and Applications

Research Group, Department of

Mathematics, Faculty of Science,

King Abdulaziz University, Jeddah,

Saudi Arabia

${ }^{2}$ Department of Mathematics,

Aligarh Muslim University, Aligarh, India

\begin{abstract}
We apply the concept of measure of noncompactness to study the existence of solution of second order differential equations with initial conditions in the sequence space $n(\phi)$.

MSC: Primary 47H09; 47H10; secondary 34A34

Keywords: Measure of noncompactness; Darbo's fixed point theorem; Sequence spaces; Infinite system of second order differential equations
\end{abstract}

\section{Introduction and preliminaries}

In recent years, the notion of measure of noncompactness has been effectively utilized in sequence spaces for different classes of differential equations (see [4, 5, 8, 11-15]). By applying this notion, Aghajani and Pourhadi [2] investigated the infinite system of secondorder differential equations in an $\ell_{1}$-space. Since then Mohiuddine et al. [10] and Banaś et al. [6] focused on this system in the sequence space $\ell_{p}$.

A measure of noncompactness is a nonnegative real-valued map defined on a collection of bounded subsets of a normed (metric) space which maps the class of relatively compact sets (known as kernel) to zero, while other sets are mapped to a positive value. There are several ways to define this notion on a given space. The widely used approach is the axiomatic one, introduced in [3], which is given below.

Let $\mathfrak{M}_{E}$ denote the family of all nonempty bounded subsets of a Banach space $E$ and $\mathfrak{N}_{E}$ be its subfamily consisting of all relatively compact sets. Let $B(x, r)$ denote the closed ball centered at $x$ with radius $r$ and $B_{r}=B(\theta, r)$.

We recall the following definition given in [3].

Definition 1.1 ([3, Definition 3.1.3]) A mapping $\mu: \mathfrak{M}_{E} \rightarrow \mathbb{R}^{+}$is called a measure of noncompactness (MNC for short) if

(i) $\operatorname{ker} \mu$ is nonempty and a subset of $\mathfrak{N}_{E}$.

(ii) $\mu(X) \leq \mu(Y)$ for $X \subset Y$.

(iii) $\mu(\bar{X})=\mu(X)$.

(iv) $\mu(\operatorname{Conv} X)=\mu(X)$.

(c) The Author(s) 2018. This article is distributed under the terms of the Creative Commons Attribution 4.0 International License (http://creativecommons.org/licenses/by/4.0/), which permits unrestricted use, distribution, and reproduction in any medium, provided you give appropriate credit to the original author(s) and the source, provide a link to the Creative Commons license, and indicate if changes were made. 
(v) For all $\lambda \in[0,1]$,

$$
\mu(\lambda X+(1-\lambda) Y) \leq \lambda \mu(X)+(1-\lambda) \mu(Y)
$$

(vi) If $\left(X_{n}\right)_{n \in \mathbb{N}}$ is a sequence of closed sets from $\mathfrak{M}_{E}$ satisfying

$$
X_{n+1} \subset X_{n} \quad \text { for all } n \in \mathbb{N} \text { and } \quad \mu\left(X_{n}\right) \rightarrow 0 \quad \text { as } n \rightarrow \infty,
$$

then

$$
X_{\infty}=\bigcap_{n=1}^{\infty} X_{n} \neq \varnothing
$$

Definition 1.2 ([5, Definition 3.1.3]) For a measure of noncompactness $\mu$ in $E$, the mapping $G: B \subseteq E \longrightarrow E$ is said to be a $\mu_{E}$-contraction if there exists a constant $0<k<1$ such that

$$
\mu(G(Y)) \leq k \mu(Y)
$$

for any bounded closed subset $Y \subseteq B$.

Darbo [7] used the idea of measure of noncompactness to obtain a new fixed point theorem which generalizes the Banach contraction principle and assures the existence of a fixed point concerning the so-called condensing operators.

Theorem 1.1 ([7]) Let $\complement$ be a nonempty, closed, bounded, and convex subset of a Banach space $E$, and let $\mathcal{G}: \complement \mapsto \complement$ be a continuous mapping such that there exists a constant $\theta \in$ $[0,1)$ with the property $\mu(\mathcal{G}(\complement)) \leq \theta \mu(\complement)$. Then $\mathcal{G}$ has a fixed point in $\complement$.

The following definition was given in [1] which is a generalization of Meir-Keeler contraction (MKC) given in [9].

Definition 1.3 ([1]) For an arbitrary measure of noncompactness $\mu$ on a Banach space $X$, we say that an operator $\mathfrak{T}: B \mapsto B$ is a Meir-Keeler condensing operator if for any $\epsilon>0$ there exists $\delta>0$ such that

$$
\epsilon \leq \mu(E)<\epsilon+\delta \quad \Longrightarrow \quad \mu(\mathfrak{T}(E))<\epsilon
$$

for any bounded subset $E$ of $B$; where $B$ is a nonempty subset of $X$.

Now we state the following theorem for Meir-Keeler condensing operators which will be applied in our main results.

Theorem 1.2 ([1]) Let $\mu$ be an arbitrary measure of noncompactness on a Banach space X. If $\mathfrak{T}: B \mapsto B$ is a continuous and Meir-Keeler condensing operator, then $\mathfrak{T}$ has at least one fixed point and the set of all fixed points of $\mathfrak{T}$ in $B$ is compact, where $B$ is a nonempty, bounded, closed, and convex subset of $X$. 


\section{The sequence space $n(\phi)$}

We denote by $\mathcal{C}$ the space of finite sets of distinct positive integers. For any $\sigma \in \mathcal{C}$, we define $\alpha(\sigma)=\left\{\alpha_{n}(\sigma)\right\}$ such that $\alpha_{n}(\sigma)$ is 1 if $n$ is in $\sigma$; and 0 elsewhere. Write

$$
\mathcal{C}_{r}=\left\{\sigma \in \mathcal{C}: \sum_{n=1}^{\infty} \alpha_{n}(\sigma) \leq r\right\}
$$

and define

$$
\Phi=\left\{\phi=\left(\phi_{k}\right): 0<\phi_{1} \leq \phi_{n} \leq \phi_{n+1} \text { and }(n+1) \phi_{n} \geq n \phi_{n+1}\right\}
$$

Sargent [16] defined the following sequence spaces which were further studied in [11]. Write $S(x)$ for the set of all sequences that are rearrangements of $x$. For $\phi \in \Phi$,

$$
\begin{aligned}
& m(\phi)=\left\{x=\left(x_{k}\right):\|x\|_{m(\phi)}=\sup _{r \geq 1} \sup _{\sigma \in \mathcal{C}_{r}}\left(\frac{1}{\phi_{r}} \sum_{k \in \sigma}\left|x_{k}\right|\right)<\infty\right\}, \\
& n(\phi)=\left\{x=\left(x_{k}\right):\|x\|_{n(\phi)}=\sup _{u \in S(x)}\left(\sum_{k=1}^{\infty}\left|u_{k}\right| \Delta \phi_{k}\right)<\infty\right\},
\end{aligned}
$$

where $\Delta \phi_{k}=\phi_{k}-\phi_{k-1}$. Note that, for all $n \in \mathbb{N}=\{1,2,3, \ldots\}, m(\phi)=\ell_{1}, n(\phi)=\ell_{\infty}$ if $\phi_{n}=1$; and $m(\phi)=\ell_{\infty}, n(\phi)=\ell_{1}$ if $\phi_{n}=n$.

We have the following important result.

Theorem 2.1 ([12]) For any bounded subset $\mathcal{Q}$ of $n(\phi)$, we have

$$
\chi(\mathcal{Q})=\lim _{k \rightarrow \infty} \sup _{x \in Q}\left(\sup _{u \in S(x)}\left(\sum_{n=k}^{\infty}\left|u_{n}\right| \Delta \phi_{n}\right)\right),
$$

where $\chi(Q)$ denotes the Hausdorff measure of noncompactness of the set $Q$ which is defined by

$$
\chi(Q):=\inf \left\{\epsilon>0: Q \subset \bigcup_{i=1}^{n} B\left(x_{i}, r_{i}\right), x_{i} \in X, r_{i}<\epsilon(i=1,2, \ldots)\right\} .
$$

\section{Infinite system of second order differential equations in $n(\phi)$}

We study the following infinite system:

$$
\frac{d^{2} u_{i}}{d t^{2}}=-f_{i}\left(t, u_{1}(t), u_{2}(t), u_{3}(t), \ldots\right) ; \quad u_{i}(0)=u_{i}(T)=0, t \in[0, T], i=1,2,3 \ldots
$$

Let $C(I, \mathbb{R})$ be the space of all continuous real functions on the interval $I=[a, b]$ and $C^{2}(I, \mathbb{R})$ be the class of functions with the second continuous derivative on $I$. A function $u=\left(u_{i}\right) \in C^{2}(I, \mathbb{R})$ is a solution of (3.1) if and only if $u \in C(I, \mathbb{R})$ is a solution of the system of integral equations

$$
u_{i}(t)=\int_{0}^{T} \mathfrak{G}(t, s) f_{i}(s, u(s)) d s \quad \text { for } t \in I,
$$


where $f_{i}(t, u) \in C\left(I \times \mathbb{R}^{\infty}, \mathbb{R}\right), i=1,2,3, \ldots$; and the Green's function associated with (3.1) is given by

$$
\mathfrak{G}(t, s)= \begin{cases}\frac{t}{T}(T-s), & 0 \leq t \leq s \leq T \\ \frac{s}{T}(T-t), & 0 \leq s \leq t \leq T\end{cases}
$$

From (3.2) and (3.3)

$$
u_{i}(t)=\int_{0}^{t} \frac{s}{T}(T-t) f_{i}(s, u(s)) d s+\int_{t}^{T} \frac{t}{T}(T-s) f_{i}(s, u(s)) d s .
$$

Now compute

$$
\frac{d}{d t} u_{i}(t)=-\frac{1}{T} \int_{0}^{t} s f_{i}(s, u(s)) d s+\frac{1}{T} \int_{t}^{T}(T-s) f_{i}(s, u(s)) d s .
$$

Again differentiating we get

$$
\left.\left.\frac{d^{2} u_{i}(t)}{d t^{2}}=-\frac{1}{T}\left(t f_{i}(t, u(t))\right)+\frac{1}{T}(t-T) f_{i}(t, u(t))\right)=-f_{i}(t, u(t))\right) .
$$

The solution of the infinite system (3.1) in the sequence space $\ell_{1}$ was discussed by Aghajani and Pourhadi [2] by establishing a generalization of Darbo type fixed point theorem using the concept of $\alpha$-admissibility function and Schauder's fixed point theorem. Here, we determine the solvability of system (3.1) in Banach sequence spaces $n(\phi)$. Our result is more general than that of [2].

Assume that

(i) The functions $f_{i}$ are defined on the set $I \times \mathbb{R}^{\infty}$ and take real values. The operator $f$ defined on the space $I \times n(\phi)$ into $n(\phi)$ as

$$
(t, u) \rightarrow(f u)(t)=\left(f_{1}(t, u(t)), f_{2}(t, u(t)), f_{3}(t, u(t)), \ldots\right)
$$

is such that the class of all functions $((f u)(t))_{t \in I}$ is equicontinuous at every point of the space $n(\phi)$.

(ii) The following inequality holds:

$$
\left|f_{n}\left(t, u_{1}(t), u_{2}(t), u_{3}(t), \ldots\right)\right| \leq g_{n}(t)+h_{n}(t)\left|u_{n}(t)\right| \text {, }
$$

where $g_{n}(t)$ and $h_{n}(t)$ are real functions defined and continuous on $I$ such that $\sum_{k=1}^{\infty} g_{k}(t) \Delta \phi_{k}$ converges uniformly on $I$ and the sequence $\left(h_{n}(t)\right)$ is equibounded on $I$.

Write

$$
G=\sup _{t \in I} \sum_{k=1}^{\infty} g_{k}(t) \Delta \phi_{k}
$$

and

$$
H=\sup _{n \in \mathbb{N}, t \in I} h_{n}(t)
$$


Theorem 3.1 Let conditions (i)-(ii) hold. Then system (3.1) has at least one solution $u(t)=$ $\left(u_{i}(t)\right) \in n(\phi)$ for all $t \in[0, T]$.

Proof Let $S(u(t))$ denote the set of all sequences that are rearrangements of $u(t)$. If $v(t) \in$ $S(u(t))$, then $\sum_{k=1}^{\infty}\left|v_{k}(t)\right| \Delta \phi_{k} \leq M$, where $M$ is a finite positive real number for all $u(t)=$ $\left(u_{i}(t)\right) \in n(\phi)$ for all $t \in I$. Using (3.2) and (ii), we have, for all $t \in I$,

$$
\begin{aligned}
& \|u(t)\|_{n(\phi)} \\
& =\sup _{v \in S(u(t))}\left(\sum_{k=1}^{\infty}\left|\int_{0}^{T} \mathfrak{G}(t, s) f_{k}(s, u(s)) d s\right| \Delta \phi_{k}\right) \\
& \leq \sup _{v \in S(u(t))}\left(\sum_{k=1}^{\infty} \int_{0}^{T}\left|\mathfrak{G}(t, s) f_{k}(s, u(s))\right| d s \Delta \phi_{k}\right) \\
& \leq \sup _{v \in S(u(t))}\left(\sum_{k=1}^{\infty} \int_{0}^{T}|\mathfrak{G}(t, s)|\left(g_{k}(t)+h_{k}(t)\left|v_{k}(t)\right|\right) d s \Delta \phi_{k}\right) \\
& =\sup _{v \in S(u(t))}\left(\sum_{k=1}^{\infty} \int_{0}^{T} \mathfrak{G}(t, s) g_{k}(t) \Delta \phi_{k} d s+\sum_{k=1}^{\infty} \int_{0}^{T} \mathfrak{G}(t, s)\left|v_{k}(t)\right| \Delta \phi_{k} d s\right) \\
& \leq \sup _{v \in S(u(t))}\left(\int_{0}^{T} \mathfrak{G}(t, s)\left\{\sum_{k=1}^{\infty} g_{k}(t) \Delta \phi_{k}\right\} d s+H \int_{0}^{T} \mathfrak{G}(t, s)\left\{\sum_{k=1}^{\infty}\left|u_{k}(t)\right| \Delta \phi_{k}\right\} d s\right) \\
& \leq \sup \sup _{v \in S(u(t))} \int_{0}^{T} \mathfrak{G}(t, s) d s+H \sup _{v \in S(u(t))} \int_{0}^{T} \mathfrak{G}(t, s) M d s \\
& \left.\leq \frac{G T^{2}}{8}+\frac{H M T^{2}}{8}=R,{ }^{T}\right)
\end{aligned}
$$

say.

Let $u^{0}(t)=\left(u_{i}^{0}(t)\right)$ where $u_{i}^{0}(t)=0$ for all $t \in I$.

Consider the closed ball $\bar{B}=\bar{B}\left(u^{0}, r_{1}\right)$ centered at $u^{0}$ and of radius $r_{1} \leq r$ which is of course a nonempty, bounded, closed, and convex subset of $n(\phi)$. Consider the operator $\mathcal{F}=\left(\mathcal{F}_{i}\right)$ on $C(I, \bar{B})$ defined as follows. For $t \in I$,

$$
(\mathcal{F} u)(t)=\left\{\left(\mathcal{F}_{i} u\right)(t)\right\}=\left\{\int_{0}^{T} \mathfrak{G}(t, s) f_{i}(s, u(s)) d s\right\}
$$

where $u(t)=\left(u_{i}(t)\right)$ and $u_{i}(t) \in C(I, \mathbb{R})$.

We have $(\mathcal{F} u)(t)=\left\{\left(\mathcal{F}_{i} u\right)(t)\right\} \in n(\phi)$ for each $t \in I$. Since $\left(f_{i}(t, u(t))\right) \in n(\phi)$ for each $t \in I$, we have

$$
\left.\sup _{v \in S(u(t))}\left(\sum_{k=1}^{\infty}\left|\left(\mathcal{F}_{k} u\right)(t)\right| \Delta \phi_{k}\right\} d s\right) \leq R<\infty
$$

Also since $\left(\mathcal{F}_{i} u\right)(t)$ satisfies the boundary conditions, we have

$$
\left(\mathcal{F}_{i} u\right)(0)=\int_{0}^{T} \mathfrak{G}(0, s) f_{i}(s, u(s)) d s=0
$$


and

$$
\left(\mathcal{F}_{i} u\right)(T)=\int_{0}^{T} \mathfrak{G}(T, s) f_{i}(s, u(s)) d s=0
$$

Since $\left\|(\mathcal{F} u)(t)-u^{0}(t)\right\|_{n(\phi)} \leq R, \mathcal{F}$ is an operator on $\bar{B}$.

The operator $\mathcal{F}$ is continuous on $C(I, \bar{B})$ by assumption (i). Now, we shall show that $\mathcal{F}$ is a Meir-Keeler condensing operator. For $\varepsilon>0$, we have to find $\delta>0$ such that $\varepsilon \leq \chi(\bar{B})<$ $\varepsilon+\delta \Rightarrow \chi(\mathcal{F} \bar{B})<\varepsilon$. Now

$$
\begin{aligned}
& \lim _{k \rightarrow \infty}\left\{\sup _{u(t) \in \bar{B}}\left(\sup _{v \in S(u(t))}\left(\sum_{n=k}^{\infty}\left|\int_{0}^{T} \mathfrak{G}(t, s) f_{n}(s, v(s)) d s\right| \Delta \phi_{n}\right)\right)\right\} \\
& \leq \lim _{k \rightarrow \infty}\left\{\sup _{u(t) \in \bar{B}}\left(\sup _{v \in S(u(t))}\left(\sum_{n=k}^{\infty} \int_{0}^{T}\left|\mathfrak{G}(t, s) f_{n}(s, v(s))\right| \Delta \phi_{n} d s\right)\right)\right\} \\
& \leq \lim _{k \rightarrow \infty}\left\{\sup _{u(t) \in \bar{B}}\left(\sup _{v \in S(u(t))}\left(\sum_{n=k}^{\infty} \int_{0}^{T} \mathfrak{G}(t, s) g_{n}(s) \Delta \phi_{n} d s\right)\right)\right\} \\
& +\lim _{k \rightarrow \infty}\left\{\sup _{u(t) \in \bar{B}}\left(\sup _{v \in S(u(t))}\left(\sum_{n=k}^{\infty} \int_{0}^{T} \mathfrak{G}(t, s) h_{n}(s)\left|v_{n}(s)\right| \Delta \phi_{n} d s\right)\right)\right\} \\
& \leq \lim _{k \rightarrow \infty}\left\{\sup _{u(t) \in \bar{B}}\left(\sup _{v \in S(u(t))}\left(\int_{0}^{T} \mathfrak{G}(t, s)\left(\sum_{n=k}^{\infty} g_{n}(s) \Delta \phi_{n}\right) d s\right)\right)\right\} \\
& +H \lim _{k \rightarrow \infty}\left\{\sup _{u(t) \in \bar{B}}\left(\sup _{v \in S(u(t))} \int_{0}^{T} \mathfrak{G}(t, s)\left(\sum_{n=k}^{\infty}\left|v_{n}(s)\right| \Delta \phi_{n}\right) d s\right)\right\} \\
& \leq H \chi(\bar{B}) \int_{0}^{T} \mathfrak{G}(t, s) d s \leq \frac{H T^{2}}{8} \chi(\bar{B}) .
\end{aligned}
$$

Hence $\chi(\mathcal{F} \bar{B})<\frac{H T^{2}}{8} \chi(\bar{B})<\varepsilon \Rightarrow \chi(\bar{B})<\frac{8 \varepsilon}{H T^{2}}$.

Taking $\delta=\frac{\varepsilon}{H T^{2}}\left(8-H T^{2}\right)$, we get $\varepsilon \leq \chi(\bar{B})<\varepsilon+\delta$. Therefore, $\mathcal{F}$ is a Meir-Keeler condensing operator defined on the set $\bar{B} \subset n(\phi)$. So $\mathcal{F}$ satisfies all the conditions of Theorem 1.2 which implies that $\mathcal{F}$ has a fixed point in $\bar{B}$, which is a required solution of system (3.1).

Remark 3.1 For $\phi_{n}=n$, for all $n \in \mathbb{N}$, the above result is reduced to that of Aghajani and Pourhadi [2] but our proof is quite different.

\section{Example}

In order to illustrate the above result, we provide the following example.

Example 4.1 Let us consider the system of second order differential equations

$$
-\frac{d^{2} u_{j}(t)}{d t^{2}}=\frac{\sqrt[j]{t}}{j^{4}}+\sum_{i=j}^{\infty} \frac{t \cos (t) u_{i}(t)}{i^{4}}, \quad j \in \mathbb{N}, t \in I=[0, T]
$$

Here $f_{i}\left(t, u_{1}(t), u_{2}(t), u_{3}(t), \ldots\right)=\frac{i \sqrt{t}}{j^{4}}+\sum_{i=j}^{\infty} \frac{t \cos (t) u_{i}(t)}{i^{4}}$, and so (4.1) is a special case of the considered system (3.1). Clearly $\frac{\sqrt[j]{t}}{j^{4}}$ and $\sum_{i=j}^{\infty} \frac{t \cos (t) u_{i}(t)}{i 4}$ are continuous on $I$ for each $n \in \mathbb{N}$. 
Notice that, for any $t \in I=[0, T],\left(f_{k}(t, u(t))\right) \in n(\phi)$ if $\left(u_{k}(t)\right) \in n(\phi)$. Moreover, we have

$$
\begin{aligned}
\sum_{k=1}^{\infty}\left|f_{k}(t, u(t))\right| & =\sum_{k=1}^{\infty}\left|\frac{\sqrt[k]{t}}{k^{4}}+\sum_{i=k}^{\infty} \frac{t \cos (t) u_{i}(t)}{i^{4}}\right| \\
& \leq \sum_{k=1}^{\infty} \frac{\sqrt[k]{t}}{k^{4}}+\sum_{k=1}^{\infty} \sum_{i=k}^{\infty}\left|\frac{t \cos (t) u_{i}(t)}{i^{4}}\right| \\
& \leq \frac{T \pi^{4}}{90}+\sum_{k=1}^{\infty} \sum_{i=k}^{\infty} \frac{t}{i^{4}}\left|u_{i}(t)\right| \\
& \leq \frac{T \pi^{4}}{90}+T\|u(t)\|_{n(\phi)}<\infty .
\end{aligned}
$$

We will show that assumption (i) is satisfied. Let us fix $\epsilon>0$ arbitrarily and $u(t)=\left(u_{k}(t)\right) \epsilon$ $n(\phi)$. Then, taking $v(t)=\left(v_{k}(t)\right) \in n(\phi)$ with $\|u(t)-v(t)\| \leq \delta(\epsilon):=\frac{\epsilon}{T}$, we have

$$
\begin{aligned}
|f(t, u(t))-f(t, v(t))| & =\sum_{i=j}^{\infty} \frac{t\left(u_{i}(t)-v_{i}(t)\right)}{i^{4}} \\
& \leq T\|u(t)-v(t)\|_{n(\phi)} \\
& \leq T \delta<\epsilon,
\end{aligned}
$$

which implies continuity as in assumption (i). Now, we show that assumption (ii) is satisfied.

$$
\begin{aligned}
\left|f_{j}(t, u(t))\right| & =\left|\frac{\sqrt[j]{t}}{j^{4}}+\sum_{i=j}^{\infty} \frac{t \cos (t) u_{i}(t)}{i^{4}}\right| \\
& \leq \frac{\sqrt{t}}{j^{4}}+\sum_{i=j}^{\infty} \frac{t}{i^{4}}\left|u_{i}(t)\right| \\
& \leq g_{j}(t)+h_{j}(t)\left|u_{j}(t)\right| .
\end{aligned}
$$

The function $g_{j}(t)=\frac{\sqrt{t}}{j^{4}}$ is continuous and $\sum_{j \geq 1} g_{j}(t)$ converges uniformly to $\frac{\sqrt{t} \pi^{4}}{90}$, also $h_{j}(t)=\frac{t \pi^{4}}{90}$ is continuous and the sequence $\left(h_{j}(t)\right)$ is equibounded on $I$ by $H=\frac{T \pi^{4}}{80}$. Also $\frac{H T^{2}}{8}<1$ is satisfied by taking $T=1.2$, which gives $H \approx 1.9739$ and $G \approx 1.9739$.

Thus, from Theorem 3.1, for a suitable value of $r_{1}$ (as discussed in Theorem 3.1) the operator $\mathcal{F}$ as defined in Theorem 3.1 on $\bar{B}\left(u^{0}, r_{1}\right)$ has a fixed point $u(t)=\left(\left(u_{i}(t)\right) \in n(\phi)\right.$, which is a solution of system (4.1).

\section{Funding}

This project was funded by the Deanship of Scientific Research (DSR) at King Abdulaziz University, Jeddah, under grant No. RG-18-130-37. The authors, therefore, acknowledge with thanks DSR for technical and financial support.

Competing interests

The authors declare that they have no competing interests.

Authors' contributions

The authors contributed equally and significantly in writing this paper. All authors read and approved the final manuscript. 


\section{Publisher's Note}

Springer Nature remains neutral with regard to jurisdictional claims in published maps and institutional affiliations.

Received: 13 June 2018 Accepted: 18 September 2018 Published online: 19 October 2018

\section{References}

1. Aghajani, A., Mursaleen, M., Haghighi, A.S.: A fixed point theorem for Meir-Keeler condensing operator via measure of noncompactness. Acta Math. Sci. 35B(3), 552-556 (2015)

2. Aghajani, A., Pourhadi, E.: Application of measure of noncompactness to $\ell_{1}$-solvability of infinite systems of second order differential equations. Bull. Belg. Math. Soc. Simon Stevin 22(1), 105-118 (2015)

3. Banaś, J., Goebel, K.: Measures of Noncompactness in Banach Spaces. Lecture Notes in Pure and Applied Mathematics, vol. 60. Dekker, New York (1980)

4. Banaś, J., Lecko, M.: Solvability of infinite systems of differential equations in Banach sequence spaces. J. Comput. Appl. Math. 137, 363-375 (2001)

5. Banaś, J., Mursaleen, M.: Sequence Spaces and Measure of Noncompactness with Applications to Differential and Integral Equation. Springer, India (2014)

6. Banaś, J., Mursaleen, M., Rizvi, S.M.H.: Existence of solutions to a boundary-value problem for an infinite system of differential equations. Electron. J. Differ. Equ. 2017, 262 (2017)

7. Darbo, G.: Punti uniti in transformazioni a condominio non compatto. Rend. Semin. Mat. Univ. Padova 24, 84-92 (1955)

8. Das, A., Hazarika, B., Arab, R., Mursaleen, M.: Solvability of the infinite system of integral equations in two variables in the sequence spaces $C_{0}$ and $\ell_{1}$. J. Comput. Appl. Math. 326, 183-192 (2017)

9. Meir, A., Keeler, E.: A theorem on contraction mappings. J. Math. Anal. Appl. 28, 326-329 (1969)

10. Mohiuddine, S.A., Srivastava, H.M., Alotaibi, A.: Application of measures of noncompactness to the infinite system of second-order differential equations in $\ell_{p}$ spaces. Adv. Differ. Equ. 2016, 317 (2016)

11. Mursaleen, M.: On some geometric properties of a sequence space related to $I_{p}$. Bull. Aust. Math. Soc. 67, 343-347 (2003)

12. Mursaleen, M.: Application of measure of noncompactness to infinite system of differential equations. Can. Math. Bull. 56, 388-394 (2013)

13. Mursaleen, M., Bilalov, B., Rizvi, S.M.H.: Applications of measures of noncompactness to infinite system of fractional differential equations. Filomat 31(11), 3421-3432 (2017)

14. Mursaleen, M., Mohiuddine, S.A.: Applications of measure of noncompactness to the infinite system of differential equations in $\ell_{p}$ spaces. Nonlinear Anal. 75, 2111-2115 (2012)

15. Mursaleen, M., Rizvi, S.M.H.: Solvability of infinite system of second order differential equations in $c_{0}$ and $\ell_{1}$ by Meir-Keeler condensing operator. Proc. Am. Math. Soc. 144(10), 4279-4289 (2016)

16. Sargent, W.L.C.: Some sequence spaces related to the $I_{p}$ spaces. J. Lond. Math. Soc. 35, 161-171 (1960)

\section{Submit your manuscript to a SpringerOpen ${ }^{\circ}$ journal and benefit from:}

- Convenient online submission

- Rigorous peer review

Open access: articles freely available online

- High visibility within the field

- Retaining the copyright to your article

Submit your next manuscript at $\gg$ springeropen.com 